\title{
Los primeros testimonios sobre Martin Seidel, autor de Origo et fundamenta religionis \\ christianae $^{1}$
}

\section{The earliest sources on Martin Seidel, author of Origo et fundamenta religionis christianae}

\author{
Pablo TORIBIO PÉREZ \\ Universidad de Sevilla
}

Recibido: 23-12-2014

Aceptado: 22-10-2015

\section{Resumen}

El tratado clandestino Origo et fundamenta religionis christianae ataca los fundamentos del cristianismo y propone una religión natural. Pese a que todas las copias manuscritas que lo conservan datan del siglo XVIII, se encuentran suficientes indicios que señalan al silesio Martin Seidel como su autor y documentan la existencia del texto a finales del siglo XVI o principios del XVII. Las primeras fuentes sobre Seidel lo vinculan con los antitrinitarios de Heidelberg (1570), los unitarios polacos (1580) y los “cripto-socinianos" de Altdorf (1610). En este artículo valoro dichas fuentes y corrijo a la luz de las mismas algunas afirmaciones de la crítica reciente sobre Seidel y su Origo.

Palabras clave: filosofía clandestina, reforma radical, neolatín, antitrinitarismo, socinianismo, exégesis bíblica, orígenes del cristianismo.

\footnotetext{
${ }^{1}$ Este trabajo se enmarca en el proyecto "Recepción, transmisión y tradición de la Biblia en griego y latín: edición y estudio de textos" (FFI2014-51910-P), con sede en el ILC (CSIC) y financiado por el Ministerio de Economía y Competitividad. Lo llevé a cabo gracias a mi contrato de investigación financiado por el MINECO (Formación Post-Doctoral 2013) y la Universidad de Sevilla, así como gracias a una beca de estancia post-doctoral de la Universidad de Friburgo (Suiza) y a una beca Regard del Instituto de Historia de la Reforma (Universidad de Ginebra) en verano y otoño de 2014. Agradezco sus correcciones y comentarios al profesor Francisco Socas y a los revisores anónimos.
} 


\begin{abstract}
The clandestine manuscript Origo et fundamenta religionis christianae attacks the foundations of Christianity and proposes a natural religion. Although all preserved copies date back to the 18th century, there is enough evidence pointing at the authorship of Silesian Martin Seidel, and documenting the existence of the text in the late 16th or early 17 th century. The earliest sources on Seidel associate him with the Heidelberg Anti-Trinitarians (1570), the Polish Unitarians (1580) and the Socinian community at Altdorf (1610). In this paper I examine these sources and correct in light of them some assertions made by recent scholarship on Seidel and his Origo.

Keywords: clandestine philosophy, radical reformation, neo-latin, anti-trinitarianism, Socinianism, biblical exegesis, origins of christianity.
\end{abstract}

\title{
1. Introducción: "un escrito deísta y anticristiano" de finales del siglo XVI
}

Martin Seidel es responsable de lo que se ha llamado "el caso deísta más temprano". 2 Su Origo et fundamenta religionis christianae se encuadra en el contexto de las tendencias teológicas radicales, y en particular las antitrinitarias, que se desarrollaron en el centro de Europa a finales del siglo XVI y durante el siglo XVII; su técnica discursiva, en consonancia con ese contexto, se basa fundamentalmente en la exégesis bíblica. Ahora bien, sus conclusiones resultan tan extremas que en retrospectiva histórica han llegado a percibirse como más cercanas al deísmo del siglo XVIII que a ninguna otra manifestación filosófica de su tiempo. En efecto, el texto de Origo "destruye los cimientos del cristianismo" y "establece los fundamentos de la religión natural":3 Seidel se propone probar, mediante un pormenorizado análisis de pasajes clave del Antiguo Testamento, que Jesús no pudo ser el Mesías, así como que la promesa del Mesías atañía exclusivamente al pueblo judío y tenía un carácter condicional: puesto que los judíos no cumplieron la condición de obediencia a Dios, no cabe ya que esperen Mesías alguno; a continuación, pone de relieve los aspectos a su juicio más irracionales y contradictorios de la religión cristiana (desde la impostura que para el autor supuso presentar a Jesús como si fuera el Mesías, hasta elaboraciones dogmáticas tales como el sacrificio de Cristo y la Trinidad) y alude a las causas filosóficas y políticas, no religiosas, de la expansión del cristia-

\footnotetext{
2 Mulsow, Martin - Popkin, Richard H. (eds.). Secret Conversions to Judaism in Early Modern Europe. Leiden: Brill, 2004, p. 15.

${ }^{3}$ En palabras de Socas, Francisco. «L'Origo et fundamenta religionis christianae: l'auteur, le texte et les thèmes». En: McKenna, Antony - Mothu, Alain (eds.). La philosophie clandestine à l'âge classique. Oxford - Paris: Universitas, 1997, pp. 213-222 (p. 213). Todas las traducciones son mías.
} 
nismo; por último, propone una religión cuya ética se base únicamente en el decálogo (que Seidel acepta no porque fuera revelado por Dios, sino porque, a su entender, es innato al ser humano) y cuya metafísica se limite al reconocimiento de Dios en las obras de la naturaleza. 4

La obra y el autor nunca han sido muy conocidos fuera de los círculos más especializados en historia eclesiástica. Esto queda de manifiesto por el subtítulo de su primera y hasta ahora única edición de 1836, debida a August Gfrörer: "un escrito deísta y anticristiano hasta ahora desconocido"; pocos años después Urban Heberle demostró (o más bien recordó) la autoría de Martin Seidel. 5 La obra misma todavía no es accesible en una edición crítica (el texto que ofrece Gfrörer se basa en un único manuscrito, por lo demás no identificado); deseamos que vea pronto la luz la que desde hace años prepara Francisco Socas, en la que tengo el honor de colaborar.

Los datos sobre la figura histórica de Martin Seidel son aún menos conocidos que su obra. Estas notas se proponen revisar y ordenar los datos disponibles: de este modo será posible matizar y corregir en algunos puntos la investigación sobre Seidel de las últimas décadas; igualmente podrá precisarse a partir de cuándo puede hablarse con seguridad de un texto titulado Fundamenta (o bien Origo et fundamenta) religionis christianae y con qué argumentos puede defenderse la autoría de Seidel.

Las referencias a Seidel en documentos del siglo XVI y de principios del XVII pueden agruparse en tres contextos históricos diferentes, aunque en buena medida vinculados: el episodio de los antitrinitarios de Heidelberg, la polémica de Fausto Sozzini con los "semijudaizantes" y el episodio de los "cripto-socinianos" de Altdorf.

\section{Martin Seidel y los antitrinitarios de Heidelberg (1564-1573)}

Consta por los registros de matrícula de la Universidad de Heidelberg que Martin Seidel se matriculó allí el 4 de mayo de 1564 (concretamente en el

\footnotetext{
4 Para un análisis de los contenidos del texto referimos al citado trabajo de Socas, «L'Origo», pp. 219222, así como a Schröder, Winfried. «Religionsphilosophie im 16. Jahrhundert? Martin Seidel und seine Schrift Origo et fundamenta religionis christianae». En: Mulsow, Martin (ed.). SpätrenaissancePhilosophie in Deutschland, 1570-1650. Tübingen: Niemeyer, 2009, pp. 161-172.

5 Gfrörer, August. «Origo et fundamenta religionis christianae: Eine bisher noch unbekannte deistische, antichristliche Schrift aus dem sechzehnten Jahrhundert». Zeitschrift für die historische Theologie, 6 (1), 1836, pp. 180-259; Heberle, Urban. «Über den Verfasser der antichristlichen Schrift Origo et fundamenta religionis christianae». Zeitschrift für die historische Theologie, 13 (1), 1843, pp. 175-193.
} 
Collegium Sapientiae); 6 así pues, tuvo que nacer en torno a 1545, en la ciudad silesia de Ohlau (hoy Oława, en Polonia), ciudad vecina de Breslau (hoy Wroclaw). ${ }^{7}$ Además, consta que Martin Seidel tenía un hermano, Jacob, probablemente más joven que él, pues se matriculó en la misma universidad dos años después. ${ }^{8}$ Martin Seidel fue elegido preceptor de la sexta clase del Paedagogium a finales de 1565. Esta institución se había refundado en Heidelberg precisamente ese año y estaba destinada a formar en lenguas clásicas a futuros alumnos de la universidad; constaba de seis clases, de las cuales la sexta era la básica. El Paedagogium dependía tanto de la universidad como del consejo eclesiástico de Heidelberg. ${ }^{9}$

En aquella época la ciudad de Heidelberg era de confesión reformada, que promovía activamente el príncipe elector del Palatinado, Federico III "el Piadoso" (1559-1576). En la universidad se desarrollaba una lucha latente entre los partidarios de una disciplina rigurosa, a semejanza de la Ginebra calvinista (este grupo era especialmente influyente en el consejo eclesiástico de la ciudad) y los partidarios de un régimen eclesiástico más liberal. En este contexto, en ningún modo exento de implicaciones políticas, se produjo el célebre escándalo de los antitrinitarios de Heidelberg: en 1570 se descubrió que algunos ministros eclesiásticos, muy conectados con la universidad y miembros del grupo "antidisciplinarista", profesaban creencias antitrinitarias o "arrianas" (es decir, de algún modo relativizaban la divinidad de Cristo) y que incluso se habían propuesto establecer contactos con los antitrinitarios de Transilvania y con los turcos. Los protagonistas del escándalo fueron Adam Neuser y Johann Sylvan: el primero acabó huyendo a Constantinopla, donde

\footnotetext{
6 Véase Toepke, Gustav. Die Matrikel der Universität Heidelberg von 1386 bis 1662. Zweiter Theil: von 1554 bis 1662. Heidelberg: Carl Winter, 1886, p. 33: Martinus Seidel, Silesius. En 1586 se matricularía en Heidelberg otro Martin Seidel, de Brieg (hoy Brzeg; véase Toepke, Die Matrikel, p. 131), también silesio, por tanto. No consta que hubiera ningún otro Martin Seidel matriculado en Heidelberg entre 1386 y 1662.

7 El dato de su ciudad natal se conoce por la firma de su primera carta a Fausto Sozzini, de la que se tratará en el epígrafe siguiente.

8 El 4 de abril de 1566, véase Toepke, Die Matrikel, p. 39: Jacobus Seidel, Olaviensis Silesius. Su matrícula fue gratuita. Jacob se graduó como magister artis en 1569 (Toepke, Die Matrikel, 464), se doctoró en medicina por la universidad de Basilea en 1575 y desde 1581 la enseñó y ejerció en Greifswald, ciudad de Pomerania donde murió en 1615. Para referencias bibliográfícas sobre él véase la edición electrónica de su inscripción funeraria: Herold, Jürgen - Magin, Christine. " DI 77, Greifswald, Nr. $253(\dagger) »$. En: Deutsche Inschriften Online <www.inschriften.net,urn:nbn:de:0238di077g014k0025301>. [17/12/2014].

9 Sobre la historia de esta institución véase Hautz, Johann Friedrich. Lycei Heidelbergensis origines et progressus. Heidelberg: Reichard, 1846, y del mismo autor, Die erste Gelehrtenschule reformirten Glaubensbekenntnisses in Deutschland, oder Geschichte des Pädagogiums zu Heidelberg unter dem Kurfürsten Friedrich III von der Pfalz in den Jahren 1565-1577, nach handschriftlichen bis jetzt noch nicht benutzten Quellen bearbeitet und nebst den wichtigsten Urkunden herausgegeben. Heidelberg: Groos, 1855. Para el nombramiento de Seidel como preceptor, véase Hautz, Lycei Heidelbergensis, pp. 119-120.
} 
murió convertido al Islam en 1578; Sylvan fue decapitado en la plaza del mercado de Heidelberg en diciembre de 1572. Otro ministro asociado a ellos fue Matthias Vehe, que logró a huir a Transilvania, donde con un nombre distinto (Glirius) fue responsable de la creación de un grupo "semijudaizante"; acabaría, sin embargo, volviendo a Alemania, donde murió encarcelado en 1590.10 Curt Horn, que estudió este episodio a principios del siglo pasado, conjetura que Martin Seidel pudo haber influido en la conversión de Johann Sylvan al "arrianismo"; 11 esto no pasa de conjetura, pues no se encuentran menciones a Seidel en la correspondencia conservada de ninguno de los protagonistas del escándalo, ni otras pruebas de su vinculación. 12

Se halla constancia documental de que en 1567 Seidel habría confiado al rector de aquel momento que "había un punto en la doctrina que no podía adoptar"; pese a que ya al día siguiente habría retirado su confesión, su confidente lo puso en conocimiento del consejo eclesiástico. ${ }^{13}$ Sin embargo, los problemas para Seidel no llegarían hasta un año después, en octubre de 1568, cuando el consejo eclesiástico solicitó al rector que lo destituyera de su cargo, pues profesaba creencias "arrianas" hasta el punto de negar la autoridad de todo el Nuevo Testamento; 14 ahora bien, el rector no reaccionó con la expulsión inmediata de Seidel, como equivocadamente

\footnotetext{
10 Sobre estos sucesos léase principalmente Burchill, Christopher J. The Heidelberg Antitrinitarians: Johann Sylvan, Adam Neuser, Matthias Vehe, Jacob Suter, Johann Hasler. Baden-Baden - Bouxwiller: Valentin Koerner, 1989; ahí mismo (p. 17) se menciona a Martin Seidel entre una serie de nombres relacionados con la universidad "que podrían merecer igualmente atención". Véase también Williams, George Huntston. The Radical Reformation: Third Edition, Kirksville, Missouri: Sixteenth Century Journal Publishers, 1992, pp. 1229-1236.

${ }^{11}$ Horn, Curt. «Joh. Sylvan und die Anfänge des Heidelberger Antitrinitarismus: Ein Beitrag zur pfälzischen Kirchengeschichte». Neue Heidelberger Jahrbücher, 17, 1913, pp. 219-310 (pp. 258-259).

12 Para los documentos me remito a Rott, Hans. «Neue Quellen für eine Aktenrevision des Prozesses gegen Sylvan und seine Genossen». Neues Archiv für die Geschichte der Stadt Heidelberg und der rheinischen Pfalz, 8, 1910, pp. 184-259; del mismo autor, «Neue Quellen für eine Aktenrevision des Prozesses gegen Sylvan und seine Genossen (Schluss)». Neues Archiv für die Geschichte der Stadt Heidelberg und der rheinischen Pfalz, 9, 1911, pp. 1-70; finalmente, Horn, «Joh. Sylvan».

13 Véanse los protocolos del consejo eclesiástico de Heidelberg, Kirchenrats-Protokolle v. J. 1567, f. 15, a, apud Hautz, Die erste Gelehrtenschule, p. 23: "Schon im Juli 1567 entdeckte er dem Rector Bock im Vertrauen, 'es sei ein Punkt in der Lehre, den er nicht fassen könnte', und bat ihn, es doch dem Kirchenrathe nicht anzuzeigen, kam aber den andern Tag wieder und erklärte, er habe seinem Irrthum entsagt. Gleichwohl berichtete Bock dem Kirchenrathe beides; sein Gewissen mochte ihm die Verschweigung einer solchen Entdeckung nicht gestatten".

${ }^{14}$ Annales universitatis, t. IX, p. II, f. 88, a, apud Hautz, Die erste Gelehrtenschule, 23-24: Petierat Senatus ecclesiasticus a M. D. Rectore, ut Martinus Seidelius, praeceptor sextae classis paedagogii, qui adeo Arrianismo infectus esse dicebatur, ut totius novi testamenti autoritatem in dubium vocaret, statim removeretur ab officio. Id, cum Senatui D. Rector exposuisset, placuit, ut prius quam ille removeretur diligenter examinaretur explorareturque, num ita res sese haberet, eoque cognito statueretur. Examinatio igitur et exploratio professoribus Theologis est demandata. Horn, «Joh. Sylvan», p. 259, repite la cita del pasaje con la referencia a las Actas, basándose en unos "extractos manuscritos de Rott" a los que habría tenido acceso.
} 
ha asumido la crítica de las últimas décadas, 15 sino con el encargo de una investigación al respecto a los profesores de teología. Esta investigación no debió de resultar muy desfavorable para Seidel, pues cuatro años después todavía es posible documentar su presencia como preceptor del Paedagogium.

Así pues, no se encuentran argumentos para negar que Martin Seidel se encontrara en Heidelberg en los turbulentos años entre el descubrimiento de la "conspiración" antitrinitaria (1570) y la decapitación de Johann Sylvan (1572): poco más de tres meses después de la misma, el 6 de abril de 1573, Seidel solicitó al rector, Thomas Erastus, permiso para viajar a Estrasburgo. ${ }^{16}$ Cuando ya se encontraba fuera de la ciudad, envió a un mensajero para que le pidiera a su hermano, Jacob, que le diera las gracias de su parte al consejo eclesiástico y que transmitiera que no pensaba volver a la ciudad. Seidel hizo llegar asimismo una carta para el rector y un escrito en el que explicaba los motivos de su marcha, que quería que se diese a conocer a todos los profesores. ${ }^{17}$ Los protocolos del consejo eclesiástico y las Actas de la universidad narran dos versiones complementarias de estos sucesos y un tanto confusas respecto a quién destruyó el escrito de Seidel (se dice que lo destruyó un tal Jungnitz, probablemente Johann Jungnitz, futuro profesor de lógica, ${ }^{18}$ y también que lo destruyó el propio hermano de Seidel, Jacob): una acción semejante al encubrimiento, que sería objeto de censura por parte del consejo eclesiástico.

En el escrito de Seidel se comprobaría que éste era "no sólo un arriano, sino también un judío y un epicúreo"; 19 el rector, Thomas Erastus, había cerrado de

\footnotetext{
15 A partir de Horn, «Joh. Sylvan», p. 259, cita el pasaje Dán, Robert. Matthias Vehe-Glirius: life and work of a radical antitrinitarian with his collected writings. Budapest - Leiden: Akadémiai Kiadó Brill, 1982, p. 18, n. 10, y nuevamente el mismo autor, «Martin Seidel's Origo et fundamenta religionis christianae and Simon Péchi», en Szczcuki, Lech (ed.). Socinianism and its role in the culture of the XVIth to XVIIIth centuries, Warsawa - Lodz: PWN, 1983, pp. 53-57 (p. 53), pero en ambos casos Dán reproduce el pasaje con la importante omisión de las dos últimas frases (id cum senatui... theologis est demandata). Concluye erróneamente (probablemente influido por Wundt, Daniel Ludwig. Magazin für die Kirchen- und Gelehrtengeschichte des Kurfürstenthums Pfalz: Erster Band, Heidelberg: Pfähler, 1789, p. 119) que Seidel fue expulsado de Heidelberg (Dán, Matthias Vehe, pp. 17-18, 177), y lleva al mismo error a quienes citan a partir de él: Williams, The Radical Reformation, p. 1232; Socas, «L'Origo», p. 214, Schröder, «Religionsgeschichte», p. 163, n. 9.

16 Para lo que sigue, véase Hautz, Die erste Gelehrtenschule, pp. 24-25, 56-58.

17 Dán, Matthias-Vehe, p. 18, n. 10, se hace eco de este envío al rector basándose en Wundt, Magazin, p. 119; ambos autores suponen que Seidel envió esta carta después de ser expulsado, pese a que dicha expulsión, como se ve, nunca se produjo.

18 Natural de Breslau, por tanto también silesio, se matriculó en Heidelberg hacia la misma época que Seidel, en 1563; se graduó en 1571, cuando ejercía como preceptor del Paedagogium al igual que Seidel; en los mismos registros consta más tarde como organi Aristotelei professor in hac academia: véase Toepke, Die Matrikel, pp. 30, 464 y 117 respectivamente.

19 Así dice el pasaje íntegro de los protocolos del consejo eclesiástico, Kirchenrats-Protokolle, 8. April 1573, f. 127, ab, apud Hautz, Die erste Gelehrtenschule, pp. 56-57: "Martinus Seidelius. Marius referirt, dieser hab beim Rectore Urlaub begehrt, In Meinung Strassburg zu sehen. Aber wie er bei
} 
golpe el libro al llegar "al pasaje en el que está escrito que el Hijo de Dios, nuestro Señor Jesucristo, no es el verdadero Mesías ni el liberador del género humano".20 El consejo eclesiástico interpretó por estos indicios que se trataba de los mismos pensamientos que Seidel había manifestado "cinco años antes" (es decir, en 1568, cuando habían solicitado al rector que lo destituyera de su cargo).

Habida cuenta de la breve descripción de su contenido (Jesum Christum non esse verum Messiam, nec liberatorem generis humani), este scriptum, libellus o

Heilbronn kommen, hab er einen antroffen und nunmehr her entbotten, sein Bruder sol sich gegen dem Kirchenrath bedanken, er gedenke nit wider zu kommen. Jacobo Seidelio gesagt, man verstehe, dass sein Bruder hinweg, sol Ursachen anzeigen. Sage, sei nechst Montag vom Rectore usgeschickt [sic] worden; sei er Ins Pädagogium gangen und sein Bruder gesucht, aber nit funden; hab er an sein statt Classen versehen; hab Rector Univ. gesagt, hab etwas heut gesehen, wolt lieber etwas anderst gesehen haben; hab ihm ein Brief gezeigt, dorin [sic] er sich des Guten bedankt, des Ime allhie erzeigt, und daneben beordert, man sol die schul mit einem andern versehen; die Ursachen hab er in ein scriptum verfasst, dass hab er bei Ime gehabt, aber noch nit verlesen. Habs Kirchenräthen wollen anzeigen; Jungnitius begert zu lesen, und Ime darnach neben Uberreichung desselben vermeldet, hab er solches zerrissen. Jungnitius hab fragmenta, so viel Ime Jungnitius zu vestehen geben, sei es aber dasjenig, das vor fünf Jaren auch mit Ime gehandelt worden. Jungnitio gesagt, man verstehe, dass er das Scriptum Seidelii soll gehabt haben, hett Im gepürt [?], solches In Kirchenrat zu geben. Sagt, Seidelii Bruder habs zerrissen und nit gelesen; sei darinnen, dass er nit allein ein Arianer, sondern auch ein Jud und Epicurer; D. Erastus hatt nichts wollen damit zu thun haben. Hatt dem Seidelio gesagt, er mögs zerreissen oder nit thun, was er woll. Soll sehen dass er die Fragmenta wider zusammen bring". En las transcripciones, sólo son mías las adiciones entre corchetes angulares o cuadrangulares.

20 Así dice el pasaje íntegro de las Actas de la universidad de Heidelberg, Annales universitatis, t. X, f. 17, b, apud Hautz, Die erste Gelehrtenschule, pp. 57-58: Mense Aprili 1573 Cum Magnif. Dom. Rector (Erastus), cur Senatum convocasset, paucis exposuisset, capita dicebat, de quibus nunc agendum est; quaedam deliberatione opus habent, quaedam vero tantum narratione. Et primo quidem ab eo capite, quod in narrando tantum consistebat, initium faciens indicabat, ante paucos dies tabellarium quendam sibi attulisse literas cum libello manuscripto, inscripto Rectori et Professoribus Universitatis. Quos [sic] cum inter prandendum accepisset, jussisse dici tabellario, se ei responsum dare tam cito non posse, quod literae non ad se solum, sed ad totam Universitatem spectent ideoque aliqui vocandi sint, qui reserationi illarum intersint. Finito prandio cum forte domo sua exiisset, tabellarium adhuc ante fores exspectasse et rogasse, ne se diu detinere vellet, ut qui viatico destitueretur. Se igitur rursum ingressum domum et adhibito Doctore Blarero (Alberto Blarero Constantiensi, Medicinae Professore), qui forte adhuc in aedibus fuerat, reserasse literas a Martino Seidelio Silesio, qui in Paedagogio Praeceptorem egerat per multos annos. Inter alia autem, haec, inquit ille, scripsi, ut intelligeretis, me non esse rediturum, petens praeterea, ut libellus suus a Senatu Universitatis legatur, et ubi lectus sit, communicetur etiam Senatui Ecclesiastico. Dom. Rector lectis, dicebat, a se et a Blatero literis et inspecto etiam hinc inde nullo ordine libello, cum in eum locum incidissent, ubi scribitur, filium Dei, Dominum nostrum Jesum Christum, non esse verum Messiam, nec liberatorem generis humani, etc., se quasi extra se raptos clausisse librum nec voluisse legendo ulterius progredi, et indignum judicasse libellum istum, qui vel Senatui exhibeatur, vel a quoque legatur. Itaque dimisso tabellario absque responso, vocasse se, dicebat D. Rector, ad se fratrem dicti Martini, qui vices secundi Praeceptoris in Domo Dionysiana agit, eique librum dedisse, ut eum porro Senatui Ecclesiastico traderet. Dictis sententiis placuit, ut Magistro Jacobo Seidelio, fratri eius, qui blasphemum istum libellum, de quo mentio est facta, ad Dom. Rectorem miserat, indicetur, ut eundem Senatui Ecclesiastico offerat. 
liber enviado en 1573 por Seidel a la universidad y al consejo eclesiástico de Heidelberg se ocupaba en esencia del mismo tema que el texto que hoy conocemos como Origo et fundamenta religionis christianae. Dos años más tarde, en el proceso formado contra Thomas Erastus (1524-1583) por su supuesta implicación con los antitrinitarios en el pasado, saldría de nuevo a relucir el incidente de Seidel (se acusaba a Erastus de haberlo encubierto) y se describiría este escrito suyo como liber contra Christianismum conscriptus. Ésa es la última información sobre Martin Seidel procedente de Heidelberg. ${ }^{21}$

Antes de cerrar este epígrafe conviene que examinemos otro dato que se transmite equivocadamente sobre la estancia de Seidel en la ciudad del Palatinado. Según Robert Dán, el teólogo calvinista Zacharias Ursinus (1534-1583), de origen silesio y activo en Heidelberg, se habría referido a Seidel como Seidelus quidam latro Silesius, cloaca diabolorum, dedecus nostrae gentis, 22 en una carta escrita en enero de 1573.23 Ahora bien, cuando se lee el pasaje íntegro puede comprobarse, en primer lugar, un error de transcripción: no se lee Seidelus ni Seidelius, sino Seidlicius, que se corresponde perfectamente con la latinización del apellido Seidlitz, suficientemente documentado en alemán; en segundo lugar, los insultos de Ursinus están motivados por el asesinato del que acusa al tal Seidlicius. ${ }^{24}$ En definitiva, en ningún modo puede concluirse por este pasaje que tales insultos tuvieran una motivación religiosa, ni mucho menos que se tratase de Martin Seidel, quien

21 Véase la transcripción de Rott, «Neue Quellen (Schluss)», pp. 13-14: Praeterea et illud S[uam] C[elsitudinem] non parum movit, quod cum Martinus Seidelius, qui quoque Arianus fuit, hinc se subduxisset et librum contra Christianismum conscriptum ad D. Erastum, qui illius anni rector esset, misisset eique significasse, se et senatui ecclesiastico et academiae librum mittere, cum utrisque servierit, id non fecerit Erastus, sed fratri Seidelii dederit et ut librum vel laceraret vel combureret suaserit, quod etiam factum est; cum haec res digna fuerit, quam pro munere, quod sustinebat, et fide, qua et C[elsitudini] S[uae] et academiae obstrictus erat, vel ad S[uam] C[elsitudinem] vel ad academiam vel senatum ecclesiasticum referret. Sobre la actividad en Heidelberg del médico Thomas Erastus, su implicación en el escándalo de los antitrinitarios y en las tensiones de la política universitaria, véase Gunnoe Jr., Charles D. Thomas Erastus and the Palatinate: A Renaissance Physician in the Second Reformation. Leiden - Boston: Brill, 2011; se menciona ahí muy brevemente (p. 246) el episodio de Seidel.

22 Citado así por Dán, «Martin Seidel’s Origo», p. 53, y a partir de él por Socas, «L'Origo», p. 214.

${ }^{23}$ El pasaje correspondiente dice así, apud Rott, Hans. «Briefe des Heidelberger Theologen Zacharias Ursinus aus Heidelberg und Neustadt a. H.». Neue Heidelberger Jahrbücher, 14, 1905, pp. 39-172 (p. 90): Scholasticum Mekelburgensem nobilem sceleratissime hic nuper interficiebat Seidlicius quidam latro Silesius, cloaca diabolorum, dedecus nostrae gentis. Cyclopum et Hippocentaurorum quorundam opera evasit aegre ferente principe. Haud dubie deus illum ad supplicium trahet. De Sylvano sumtum esse supplicium puto me scripisse. Non desunt, qui cavillentur. Dán, «Martin Seidel's Origo», p. 53, también remite a esta edición de Rott.

${ }^{24} \mathrm{La}$ víctima del asesinato fue el joven noble Hermann Moltzan, muerto el 1 de noviembre de 1572 (Rott, «Briefe», 91, escribe "noviembre de 1573", una errata, pues el mismo autor fecha la carta, en razón de su contenido, en enero de ese mismo año). Véase Toepke, Die Matrikel, p. 84, n. 2. 
tres meses después de la escritura de esta carta, como hemos visto, seguía dando clases normalmente en el Paedagogium.

Nuestro autor, pues, se marchó de Heidelberg, donde había pasado nueve años, en abril de 1573, dejando tras de sí el escándalo de un "escrito blasfemo". La siguiente noticia sobre él viene de Polonia, aproximadamente una década más tarde y de su propia mano.

\section{Martin Seidel, Fausto Sozzini y los "semijudaizantes" (ca. 1584)}

En las obras completas de Fausto Sozzini (1539-1604) se encuentra un breve "escrito contra los semijudaizantes" (adversus semijudaizantes scriptum); este texto sirve como introducción para un intercambio epistolar no fechado, consistente en tres cartas con sus respuestas pasadas entre Martin Seidel, que se dirige a la comunidad unitaria de Cracovia, y el propio Fausto Sozzini, que responde en nombre de dicha comunidad. 25

Sozzini llama "semijudaizantes" a quienes "sin haber nacido hebreos ni guardar, por lo demás, la ley de Moisés en todo, sin embargo se niegan a reconocer a Jesús de Nazaret como su salvador, es decir, a tenerle fe, y directamente rehúsan obedecerle en la esperanza de la salvación eterna, porque dicen que él no es aquel Mesías o Rey que fue prometido por Dios a través de los profetas". 26 En el último tercio del siglo XVI se localizaban en el área de las actuales Polonia, Lituania y Transilvania, entre otras manifestaciones de la Reforma radical, una serie de corrientes religiosas llamadas "judaizantes" por sus adversarios. Estas corrientes engloban un grupo confuso y heterogéneo de diversas creencias radicales muy mal conocidas. ${ }^{27}$ La tendencia más documentada consistía en la adopción de ciertos

25 El conjunto (tratado y cartas) se publicó por primera vez al comienzo de una miscelánea póstuma de las obras de Sozzini, Fausto. Fausti Socini miscellanea, hoc est, scripta theologica seu tractatus breves de diversis materiis. Racoviae [Raków]: typis Sebastiani Sternacii, 1611, pp. 3-50. Aparece reimpreso al final de sus obras completas: Fausti Socini Senensis operum tomus alter, continens ejusdem scripta polemica. Irenopoli, post annum Domini 1656 (=Ámsterdam, 1668, según Sand, Christoph. Bibliotheca Anti-Trinitariorum. Freistadii [=Ámsterdam]: apud Johannem Aconium, 1684 p. 79 ), pp. 804-812. Me remito a esta última edición.

26 Sozzini, Opera, p. 804a.

27 Véase Szczucki, Lech. «L'antitrinitarismo in Polonia: tendenze della ricerca e prospettive». En: Movimenti ereticali in Italia e in Polonia nei secoli XVI-XVII: Atti del Convegno Italo-Polaco, Firenze, 22-24 settembre 1971. Firenze: Istituto Nazionale di Studi sul Rinascimento, [1974], pp. 541 (pp. 13-15); Dán, Robert. «Judaizare: the career of a term», en Dán, Robert - Pirnát, Antal (eds.). Antitrinitarianism in the second half of the 16th century, Budapest - Leiden: Akadémiai Kiadó - Brill, 1982, pp. 25-34 (pp. 30-31); Pietrzyk, Zdzisław. «Les judaïsants en Pologne dans la seconde moitié du XVIe siècle». Cahiers du Centre d'études juives, 2, 1991, pp. 25-37 (pp. 30-32); Fleischmann, Stefan. Szymon Budny: ein theologisches Portrait des polnisch-weißrussischen Humanisten und Unitariers (ca. 1530-1593). Köln: Böhlau, 2006, pp. 43-44. 
ritos judíos (observancia del sabbat, circuncisión) y en la negación de que deba adorarse e invocarse a Cristo, en la idea que su reino no entrará en vigor hasta la segunda venida; con este grupo de creencias se asocian nombres como los de Ferenc Dávid (ca. 1510-1579), el ya mencionado Matthias Vehe-Glirius, o Szymon Budny (ca.1530-1593). Más radical resulta el grupo que el teólogo antitrinitario Marcin Czechowic (1532-1613) designa como "pagano-judíos", cuyos miembros negaban que Cristo fuera el Mesías, así como la autoridad del Nuevo Testamento, y sólo aceptaban el decálogo como norma religiosa. Czechowic considera como cabecilla de este grupo a Daniel Bieliński, a quien alguna fuente documental presenta como vinculado a la región de Silesia; ${ }^{28}$ se dispone de muy pocos datos más sobre él. La coincidencia de esta descripción con las ideas del propio Seidel resulta enormemente llamativa.

Con esta compleja situación religiosa de fondo, Sozzini había llegado desde Basilea hasta Cluj (Transilvania) en 1578, llamado por el también antitrinitario Giorgio Biandrata ( $c a .1515-1588$ ) para que solucionase la heterodoxia del obispo local, el referido Ferenc Dávid. Con Dávid colaboraba estrechamente Matthias Vehe-Glirius, a quien ya se ha mencionado entre los antitrinitarios de Heidelberg. ${ }^{29}$

Sozzini fracasó en su misión de disuadir al obispo de Cluj de su postura contraria a la adoración de Cristo; Dávid murió en la cárcel en 1579 y Sozzini marchó a Polonia y se instaló en la ciudad de Cracovia, donde ejerció una creciente influencia sobre la comunidad antitrinitaria local, la ecclesia reformata minor, hasta su muerte en 1604. Durante la última parte de su vida el teólogo sienés protagonizó otros debates en defensa de la "ortodoxia" antitrinitaria, notablemente con Christian Francken, que también rechazaba la adoración de Cristo. ${ }^{30}$ El debate con Francken precede inmediatamente al intercambio epistolar con Seidel en la edición de las obras completas de Sozzini; dado que dicho debate tuvo lugar el catorce de marzo de 1584, suele situarse el intercambio con Seidel hacia esa época, aunque no se encuentran razones concluyentes para ello. ${ }^{31}$ En todo caso, puesto que Sozzini res-

\footnotetext{
28 Véase Chmaj, Ludwik. Bracia Polscy: Ludzie, idee, wplywy. Warszawa: PWN, 1957, pp. 25-29.

29 Vehe-Glirius es responsable del grupo transilvano conocido como "sabatarianos", cristianos que observaban el sabbat y otros ritos de la ley judaica. Viajó a Polonia a finales de la década de 1570 o principios de la de 1580 y permaneció allí hasta 1588 , por tanto durante el período en el que Seidel se dirigió a la comunidad sociniana de Cracovia, como en seguida veremos. En 1581 fue huésped de Szymon Budny, quien como Sozzini negaba la preexistencia de Cristo y como Dávid negaba también su adoración. Véase Burchill, The Heidelberg Antitrinitarians, pp. 157-209 (p. 161).

${ }^{30}$ Sobre el radicalismo de Francken, así como sobre el contexto ideológico de Transilvania en la época y los aspectos que nos ocupan, véase Simon, József. Die Religionsphilosophie Christian Franckens, 1552-1610?: Atheismus und radikale Reformation im frühneuzeitlichen Ostmitteleuropa. Wiesbaden: Harrassowitz, 2008.

31 Esta datación tentativa se debe a Chmaj, Ludwik. Faust Socyn (1539-1604). Warszawa: PWN, 1963, pp. 233-234 (debo la noticia al Dr. Kestutis Daugirdas, que amablemente ha hecho accesibles para mí los pasajes pertinentes de este autor polaco). Pietrzyk, «Les judaïsants en Pologne», p. 32,
} 
ponde a Seidel en nombre de la comunidad de Cracovia, el intercambio tuvo que producirse necesariamente después del establecimiento del primero en Polonia, a partir de 1579, y antes de su muerte en 1604.32

En su primera carta, ${ }^{33}$ Seidel se dirige a los unitarios cracovienses para pedirles un puesto de maestro de escuela entre ellos, aunque dicha intención no se revela hasta el final, al menos en el estado en que se ha transmitido la carta. Primero, Seidel describe la contradicción entre el Antiguo y el Nuevo Testamento en cuanto a la naturaleza del Mesías prometido a los judíos y declara que, ante dicha contradicción, él prefiere quedarse con los profetas y entender por tanto que el Mesías debía haber sido un rey terreno, al que Dios nunca envió ni enviará, debido a la desobediencia del pueblo judío. En cuanto a la validez de las razones que, según Seidel, esgrimen los cristianos para probar que su religión es la verdadera (los milagros de Jesús y sus discípulos, sus martirios, la expansión milagrosa del cristianismo y su perduración, los "preceptos santísimos" que en esta religión se contienen y sobre todo la promesa de la vida eterna), escribe Seidel que mostraría su debilidad si supiese que no iba a ofender a los unitarios, pero que supone que, como el resto de los cristianos, éstos no se tomarán a bien "que alguien discuta los fundamentos de la religión cristiana" (si quis de fundamentis Christianae religionis disputet). A continuación Seidel resume los fundamentos de su propia religión y alude a un escrito suyo en el que desarrolla sus principios en detalle y que, según dice, está en poder de los unitarios (ut sciatis, cujus sim religionis, quamvis id scripto meo, quod habetis, ostenderim, tamen id breviter hic repetam). Repite que la doctrina del Mesías no atañe a su religión y que ésta sólo consiste en el decálogo, "que es la voluntad eterna e inmutable de Dios" (verus autem cultus Dei, quem meam religionem appello, est Decalogus, qui est aeterna et immutabilis voluntas Dei), y ello no porque fuera revelado al pueblo judío "por medio de una voz que sonó desde el cielo", sino porque "es innato a la mente a través de la creación" (per creationem insitus est menti meae). El "decálogo vocal”, es decir, el que Dios reveló a los judíos, puede emplearse instrumentalmente para aclarar el "decálogo innato", puesto que éste, "por la corrupción de la naturaleza humana y las malas costumbres, se ha oscurecido en alguna medida" (quia autem insitus Decalogus, per corruptionem naturae humanae, et pravis consuetudinibus aliqua ex parte obscuratus est, ideo ad illustrandum eum, adhibeo vocalem decalogum).

Es al final de la carta, tal y como se nos ha conservado, donde Seidel revela su intención de conseguir un puesto escolar entre los unitarios y donde aporta algunos

asume sin más esta datación, así como la conjetura de que Seidel fue huésped de Alexander Witrelin en Lublin.

32 Heberle, «Über den Verfasser», p. 189, estrecha más los termini post y ante quem y propone el período 1587-1598 para el intercambio epistolar, teniendo en cuenta que Sozzini se ausentó frecuentemente de Cracovia antes y después de esos años.

33 Sozzini, Opera, pp. 806a-807a. 
datos biográficos. ${ }^{34}$ Debe resaltarse que Seidel afirma ahí que "no conoce a nadie de su opinión" y que se dirige a los unitarios porque éstos constituyen "la secta que más se ha acercado a la verdad": quizás estas afirmaciones descartan su asociación con los grupos "semijudaizantes" mencionados más arriba, en particular con Daniel Bieliński. Estas declaraciones y la ausencia de otros testimonios inducen a pensar que la inclusión de Seidel en un grupo de "semijudaizantes" obedece a una construcción teórica de Sozzini y de los teólogos posteriores, pero que en principio no debe entenderse como una pertenencia real a ningún grupo disidente determinado. También resulta interesante cuando escribe que "durante algunos años había intentado apartar de la idolatría a sus compatriotas alemanes con sus escritos, e incluso conversando con ellos, con gran peligro de su vida", pero que al no tener éxito en Alemania, se había dirigido a los unitarios de Cracovia "directamente". Por las Actas de la Universidad de Heidelberg aducidas arriba se sabe que Seidel hizo un tímido amago de difusión de sus ideas en Alemania en 1567 y con decisión y por escrito a partir de 1573. Quizás el hecho de que Seidel cifre la duración de sus esfuerzos proselitistas en Alemania mediante la expresión per aliquot annos, "durante algunos años", invite a considerar que su intercambio epistolar con Sozzini fuera más bien en los comienzos del período delimitado más arriba (1579-1604); en ese sentido, resultaría plausible la conjetura de Chmaj para 1584.

La respuesta de Sozzini, así como la segunda carta de Seidel y la siguiente respuesta del toscano, se detienen en aspectos exegéticos: Sozzini se esfuerza por defender la identificación del Mesías prometido en el Antiguo Testamento con el Jesús del Nuevo, y Seidel se afana por rebatirla. ${ }^{35} \mathrm{Al}$ final de su tercera y última

\footnotetext{
34 Sozzini, Opera, pp. 806b-807a: Haec est mea sententia de Messia, seu Rege illo promisso, et haec est mea Religio, quam coram vobis ingenue profiteor. Neque me ideo huc contuli, quod de hac mea religione dubitarem, tantum abest, ut eam mutare voluerim. Cum sciam eam solam esse verissimam et certissimam; sed hanc ob caussam, sicut vobis initio dixi, quod de Germanis meis desperarim, quos hactenus per aliquot annos scriptis meis ab idololatria avocare conatus sum, et quidem tanto studio, ut postremo, etiam, cum praesenti periculo vitae meae, voluerim cum ipsis hac de re colloqui. Quod quia renuerunt, nihil amplius facere potui. Atque ideo inde discessi et recta ad vos profectus sum, utpote ad eos, qui propius, quam aliae sectae, ad veritatem accesserunt. Nam cum neminem sciam meae sententiae, neque quisquam hanc sententiam suscipere velit, ego vero sciam quod haec sit ipsa veritas Dei, atque ideo ab hac veritate Dei bona conscientia discedere non possim, et mihi tamen cum hominibus vivendum sit, malo me conjungere melioribus, quam deterioribus. Et quia adulti nolunt a me doceri de religione, ideo pueros docebo litteras, item necessarias linguas et artes. Non itaque ambio munus ecclesiasticum, non enim volo turbare institutum vestrum, sed peto scholam aut paedagogiam aliquam. Quid autem hac in re sit animi vestri, et an locum apud vos habere possim, id mihi ut significetis, peto. Martinus Sejdelius, Olaviensis Silesius.

35 Sozzini, Opera, pp. 807a-810b. Sobre la exégesis veterotestamentaria de Sozzini en general véase Nellen, H. J. M. «Growing tensions between Church doctrines and critical exegesis of the Old Testament». En: Saebø, Magne (ed.). Hebrew Bible / Old Testament: The history of its interpretation. Volume II: From the Renaissance to the Enlightenment. Göttingen: Vandenhoeck und Ruprecht, 2008, pp. 803-808. Es notable la absoluta ausencia de Seidel en esta voluminosa obra de referencia sobre interpretación del Antiguo Testamento en la Edad Moderna.
} 
carta, Seidel se despide de los unitarios, pues ha llegado a la conclusión, dice, de que éstos no van a aceptarlo entre ellos si no se adhiere a su parecer sobre el Mesías, es decir, si no se hace cristiano. Aduce la insuficiencia de sus recursos económicos para quedarse más tiempo y se despide cordialmente de los unitarios y en concreto del "señor Alejandro", al que agradece por su hospitalidad. ${ }^{36}$ Ludwik Chmaj identificó a este Alejandro con el antitrinitario Alexander Witrelin y dedujo por ello que Seidel escribió desde Lublin, pero no exhibe las razones de su deducción. ${ }^{37}$

Por último, parece digno de resaltarse que Seidel describe su discurso sobre la religión cristiana como un "hablar de fundamentos": dice en su primera carta que a los cristianos "no les gusta oír cuando alguien discute los fundamentos" de su religión y al despedirse de los unitarios de Cracovia escribe que él intenta sacar a la gente de la idolatría "basándose en fundamentos distintos" a los de ellos. No puede descartarse que Seidel fuera ya entonces autor de un texto titulado Fundamenta religionis christianae, que bien podría ser el "escrito" que, según él mismo refiere en su primera carta, los unitarios de Cracovia tenían en sus manos.

Éstas son las últimas noticias que documentan la existencia histórica de Martin Seidel y que al mismo tiempo conservan sus creencias de la manera en que él mismo las declaró. El contacto epistolar con Sozzini y la publicación de estas cartas entre las obras del teólogo sienés garantizaron que su recuerdo perdurase entre los historiadores eclesiásticos protestantes, sobre todo entre los socinianos. Las pruebas de la recepción de Seidel entre éstos, así como el primer testimonio de la existencia de un texto titulado Fundamenta (o bien Origo et fundamenta) religionis christianae, proceden de Alemania en las primeras décadas del siglo XVII, como se verá a continuación.

\footnotetext{
36 Sozzini, Opera, pp. 810b-811b: Quamvis autem mihi hactenus ad meam petitionem non responderitis, facile tamen possum colligere responsum tale futurum, me non posse apud vos habere locum, nisi in doctrina de Messia vobiscum consentiam, quod cum certo sciam, et multo certius sciam, sententiam meam hac in re esse certissimam, atque ideo bona conscientia eam non possim abnegare, ideo cogor hinc discedere, praesertim cum sim destitutus sumptibus et nolim diutius gravare benefactores meos. Gratias itaque vobis ago pro humanitate et benevolentia vestra quam mihi ostendistis, nominatim vero et praecipue gratias ago Domino Alexandro pro beneficiis suis in me peregrinum collatis, utinam aliquando ei gratiam referre possim, sed quia jam non possum, rogo Deum, ut ei centuplum reddat, quod in me boni contulit. Atque vos Deo opti<mo $>$ maximo commendo, et quamvis vobiscum in doctrina de Messia non consentiam, tamen optarem ut omnes Christiani vestram sententiam amplecterentur, et ab idololatria sua discederent, a qua idololatria etiam ego ipsos conor abstrahere, sed ex aliis fundamentis quam vos facitis. Valete. Martinus Sejdelius.

37 Véase Chmaj, Faust Socyn, pp. 233-234. El conocimiento sobre Alexander Witrelin es exiguo; entre otros pocos datos se sabe que era pastor en Pinczow en 1555, que participó en el Sínodo de Lublin en 1579 y que un manuscrito suyo contra Christian Francken estaba fechado en mayo de 1584 (Sand, Bibliotheca, pp. 46-47, y Bock, Friedrich Samuel. Historia Antitrinitariorum, maxime Socinianismi et Socinianorum, tomus I, pars II. Regiomonti [Königsberg] et Lipsiae: impensis Gottl. Lebr. Hartungii, pp. 983-984).
} 


\section{La recepción de Seidel entre los "cripto-socinianos" de Altdorf (1605-1617)}

En la luterana Academia de Altdorf (ciudad muy cercana a Núremberg, llamada hoy Altdorf bei Nürnberg) se produjo en la segunda década del siglo XVII otro escándalo semejante al que se había producido en Heidelberg cuarenta años antes: entre los estudiantes se había formado durante años una comunidad de conversos en secreto al socinianismo, o "cripto-socinianos". ${ }^{38}$ La labor de proselitismo se remontaba al profesor de medicina y filosofia Ernst Soner (1572-1612), convertido por dos socinianos (Christoph Ostorodt y Andreas Voidovius o Wojdowski) durante una estancia de estudios en Leiden (1597); después de su conversión, Soner había ocupado una cátedra de filosofía en Altdorf desde 1605 hasta su prematura muerte en 1612 , sin que surgiera entonces la menor sospecha sobre su ortodoxia religiosa. Es de destacar que Soner fue maestro de Johann Crell (1590-1633), el influyente teólogo sociniano, que abandonó Alemania pocos días después de la muerte de aquél. El grupo "cripto-sociniano" creció en torno a otro alumno de Soner, Martin Ruar (1588-1657), que extendió su labor proselitista hasta Estrasburgo. Pero la comunidad secreta de Altdorf fue descubierta en 1615 debido a la indiscreción de algunos de sus miembros, que igualmente habían llevado su "misión" a otras universidades luteranas (Jena, Wittenberg, Helmstedt, Rostock): los estudiantes que no lograron escapar (el propio Ruar sí lo consiguió) fueron arrestados y se les obligó a abjurar del socinianismo. En 1616 se llevó a cabo en la plaza del mercado de Altdorf una quema pública de libros confiscados a los "cripto-socinianos", entre los que se encontraba un manuscrito titulado Orthodoxum fundamentum religionis christianae, que Zeltner no duda en atribuir a Martin Seidel. ${ }^{39}$

Es preciso aclarar en seguida que no se encuentra ningún fundamento para conjeturar que Martin Seidel se encontrara físicamente presente en Altdorf, mucho menos en calidad de estudiante:40 si Seidel aún vivía en la época de estos sucesos,

\footnotetext{
38 A estos hechos está dedicada la voluminosa obra de Zeltner, Gustav Georg. Historia crypto-socinismi [sic], Altorfinae quondam academiae infesti, arcana, ex documentis maximam partem manuscriptis ita adornata, ut cum historiae illorum hominum illustrandae tum dogmatibus in universum refellendis inservire possit. Accesserunt praeter alia Valentini Smalcii diarium vitae ex autographo et Martini Ruari epistolarum centuriae duae, hactenus rarius apparentes, hic vero etiam notis idoneis illustratae, I-II. Lipsiae: apud Jo. Frid. Gleditschii B. Fil., 1729. Se encuentra un resumen en Wilbur, Earl Morse. «Socinian propaganda in Germany three hundred years ago: crypto-Socinianism at Altdorf», Proceedings of the Unitarian Historical Society 3 (1), 1933, pp. 22-41. Se cuenta ahora también con la contribución de Marti, Hanspeter - Marti-Weissenbach, Karin (eds.). Nürnbergs Hochschule in Altdorf: Beiträge zur frühneuzeitlichen Wissenschafts- und Bildungsgeschichte. Köln: Böhlau, 2014. La Academia de Altdorf alcanzó el rango de universidad en 1623 (Nürnbergische Universität Altdorf) y existió hasta 1809.

39 Zeltner, Historia, I, p. 495, donde reproduce una selección de los libros confíscados a partir de documentos de la universidad.

40 Como escribe sin embargo Wollgast, Siegfried. Philosophie in Deutschland zwischen Reformation und Aufklärung (2. Auflage). Berlin: Akademie Verlag, 1993, p. 377. A partir de Wollgast repite sin
} 
tendría ya más de sesenta años (dedujimos más arriba, a partir de la fecha de su matrícula en Heidelberg en 1564, que debió de nacer en torno a 1545). En cambio, sí era alumno de Altdorf y "cripto-sociniano" otro Seidel, de nombre Georg, natural de Greifswald, en Pomerania. ${ }^{41}$ Zeltner conjetura que este Georg, por edad y por su ciudad natal, bien podría ser hijo de Jacob Seidel, el hermano de Martin, que como hemos visto acabó establecido como profesor de medicina en aquella ciudad. Si esto fuera así, Georg sería sobrino de nuestro Seidel; así lo asume Zeltner, que supone también que "quizás de su tío bebió Georg los principios de su herejía". Georg se marchó de Altdorf poco antes del escándalo (1615) y se estableció en Polonia, donde Zeltner asume que permaneció hasta su muerte. ${ }^{42}$

Uno de los principales estudiantes de la comunidad "cripto-sociniana" fue Johann Vogel, latinizado Avitus (1589-1663), que antes del escándalo había viajado con el propio Ruar por Alemania y por Polonia, y allí había entrado en contacto directo con círculos socinianos locales. En 1615 Vogel mantuvo en Wittenberg una disputatio académica con el profesor de teología Jacob Martini (1570-1649);43 la disputatio trataba sobre el Mesías y Vogel adujo una serie de argumentos que había extraído de un manuscrito de Martin Seidel, como el propio Vogel narra en una noticia que reproduciremos a continuación. Jacob Martini le pidió que le entregara el manuscrito, e incluyó una extensa refutación del mismo en el tercer tomo de sus De tribus Elohim, publicado en 1619.44 En cuanto a Vogel, cuando más adelante fue descubierta su asociación con los socinianos fue arrestado y llevado a Altdorf, donde sufrió prisión junto con otros compañeros. En 1617 abjuró oficialmente de sus creencias antitrinitarias.

La disputatio celebrada en Wittenberg en 1615 entre Vogel y Jacob Martini constituye el momento clave en la historia de la recepción del Origo; como se acaba de decir, este ejercicio académico ofreció la ocasión para la escritura de la mayor

más el dato espurio Schmeisser, Martin. Sozinianische Bekenntnisschriften: der Rakówer Katechismus des Valentin Schmalz (1608) und der sogennante Soner-Katechismus. Berlin: Akademie Verlag, 2012, p. 57, y quizás, aunque no aporta su fuente, también Schröder, «Religionsphilosophie», p. 164, que se pregunta si Seidel habría llegado a conocer personalmente a Soner. Desvincula a Seidel de los "cripto-socinianos" Achermann, Eric. «Ratio und oratio mentalis: Zum Verhältnis von Aristotelismus und Sozinianismus am Beispiel der Philosophie Ernst Soners». En: Marti y Marti-Weissenbach (eds.). Nürnbergs Hochschule in Altdorf, pp. 98-157 (p. 113, n. 55).

41 Matriculado el 27 de octubre de 1610 como Georgius Seidelius Gryphiswaldensis Pom., véase Steinmeyer, Elias von. Matrikeln Fränkischer Schulen, vierte Reihe, erster Band: Die Matrikel der Universität Altdorf. Erster Teil. Würzburg: Stürzt, 1912, p. 113.

42 Véase Zeltner, Historia, I, pp. 335-336 (la cita literal en p. 336, en nota).

43 Martini, Jacob - Vogel, Johann. «Disputatio VII. De Messia. Probans contra Judaeos Jesum Christum Dominum nostrum esse verum Messiam. In academia Wittebergensi praeposita praeside Jacobo Martini, respondente Johanne Vogelio Noribergensi, ad diem 8 Novembris». En: Martini, Jacob. Disputationum de Messia Iudaeorum blasphemiis oppositarum decas. Wittebergae, 1616.

${ }^{44}$ Martini, Jacob. Liber tertius de tribus Elohim, oppositus judaeis et semijudaizantibus. Wittebergae: impensis Zachariae Schureri bibliopolae, in officina Johannis Richteri viduae, 1619. 
parte del Liber tertius de tribus Elohim (1619), donde Martini ofrece muy extensas citas de un manuscrito titulado Origo et fundamenta religionis christianae. Dichos extractos (prácticamente la totalidad de la primera parte de la obra tal y como hoy la conocemos) atestiguan que el tratado existía ya entonces y con ese título; de hecho, los extractos de Martini pueden considerarse una "publicación parcial" del Origo, como ha señalado Winfried Schröder, ${ }^{45}$ y constituyen el testimonio más antiguo conservado del texto de Seidel.

Tras una primera parte "dirigida contra los errores de los judíos", Martini dedica la segunda y más extensa parte de su libro a refutar "las locuras de los semijudíos" (pp. 336-752). Comienza Martini esta parte con el relato de cómo, "hace más de un año, una persona muy instruida en latín, griego y hebreo y versado no poco en la lectura de la Biblia, pero conmovido en no pequeña medida por los argumentos de los semijudíos", le presentó "un manuscrito en el que su autor, un blasfemo semijudío, intenta demostrar que el Mesías no ha venido y nunca ha de venir" y le pidió a Martini que lo refutase. Esta persona que el teólogo de Wittenberg deja sin nombrar es Johann Vogel, y la circunstancia de la entrega del manuscrito es la mencionada disputatio, como aclara el testimonio del propio Vogel que en breve se referirá. Martini se extiende a continuación (pp. 337-373) en la refutación de "la religión de los semijudíos" tal y como ésta había quedado caracterizada en el intercambio epistolar entre Seidel y Sozzini. Después de ocuparse de estas cartas, dedica el resto del libro (pp. 373-752) a refutar por extenso "un manuscrito singular" debido a "cierto anónimo" y titulado Origo et fundamenta religionis christianae. 46 No dice expresamente que éste fuera el manuscrito al que se había referido al principio (pp. 336-337), aunque ésa parece la inferencia más lógica. ${ }^{47}$ Más notable resulta que no lo atribuya a Seidel, pese a que ha estado hablando de sus cartas con Sozzini hasta ese momento: con bastante probabilidad, pues, el manuscrito en poder de Martini no llevaba nombre de autor.

Sin embargo, Johann Vogel sí afirma expresamente que el autor del manuscrito que le entregó a Martini era Martin Seidel; también afirma que llevaba el título de Fundamenta (no Origo et fundamenta) religionis christianae. Conocemos el propio relato de Vogel por sus notas manuscritas, según las transcribió casi un siglo después su nieto Nicolaus Gundling (1671-1729) en el primer volumen de Gundlingiana (1715). 48

\footnotetext{
45 Schröder, «Religionsphilosophie», p. 164, n. 15.

46 Martini, Liber tertius, p. 374, transcribe claramente el título del manuscrito, pese a lo que escribe Schröder, «Religionsphilosophie», p. 164, n. 15.

47 Cautamente lo considera una obra diferente, aunque igualmente se la atribuye a Seidel, Bock, Historia Antitrinitariorum, p. 832.

48 Gundling, Nicolaus Hieronymus. Gundlingiana, darinnen allerhand zur Jurisprudenz, Philosophie, Historie, Critic, Litteratur und übrigen Gelehrtsamkeit gehörige Sachen abgehandelt werden. Erstes Stück. Halle in Magdeburgischen: zu finden in der Rengerischen Buchhandlung, 1715, pp. 26-51, y
} 
Después de abjurar públicamente del socinianismo en 1617, Johann Vogel alcanzó prosperidad social: en 1621 fue nombrado rector de la escuela de San Egidio en Núremberg y de San Sebaldo a partir de 1633,49 cargo en el que permaneció hasta su muerte. Sin embargo, según documenta una carta del mismo año 1621, Vogel todavía desempeñó una vez más el papel de difusor del texto seideliano, si bien de forma algo reticente. La carta está dirigida a Simon Péchi, sumo canciller del príncipe de Transilvania; debido a su rareza y al interés de sus contenidos e implicaciones está reproducida por completo en nota. En todo caso, debe tomarse con las precauciones que aconseja el hecho de que sólo se conozca por una transcripción de mediados del siglo XIX; el autor del artículo, Joseph Kemény, no da información sobre el manuscrito en el que leyó esta carta, de la que sólo dice que "llegó a sus manos por azar". 50

sobre todo pp. 40-42: "Dieser mein Herr Praeses hielte mich hoch und begehrte einesmals, als ich ihm in den Genealogiis Christi etliche difficultäten wiese, welchen er nicht gewachsen ware, ich solte ihm doch sagen, woher ich allezeit pro Judaeis so gute Argumenta nähme, darauf gab ich ihm ein geschriebenes Buch, dessen Titul [sic] war: Fundamenta Religionis Christianae. Es waren darinnen alle Oerten der heiligen Schrifft so aus dem Alten Testament im Neuen angezogen waren, examinirt und [p. 41] auf sensum litteralem gezogen, also daß sie nicht bewiesen, wozu sie die Evangelisten anziehen, waren auch andere Sachen, die der Christlichen Religion zuwieder lauffen, darinnen begriffen. [...] Des Buchs Autor ist gewesen Martinus Seidelius, ein Schlesier, welcher weder ein Christ, noch Jud, noch Türck seyn wollen, sondern bloß Theologiam naturalem gehabt, und dafür gehalten, es wäre genug, wenn er nach den zehen Gebotten lebte, nicht darum, daß sie von Gott wären gegeben worden, als welche dißfals nur die Juden angiengen, deren Policey längst vergangen, sondern dieweil sie mit dem Licht der Natur überein kämen. Es wäre auch kein Messias jemahls den Juden gegeben worden, ob er gleich versprochen worden. Denn die Verheissungen von dem Messia im Alten Testa- [p. 42] ment, das nuumehro [sic] nichts als eine blosse historie wäre, wären alle conditionales gewesen, wann die Juden Gott würden gehorsam seyn; Dieweil aber diese condition nicht erfolgt, wäre auch kein Messias erfolgt, und was wir von dem Jesu von Nazareth sagten, oder glaubten (Gott verzeihe es mir, daß ich es erzehle) wäre alles nichtig, und darum unterstunde er sich alle dicta Veteris Testamenti mit einem solchen Strategemate zueludiren, und die Evangelisten dahin zubringen, als ob sie, sonderlich in den Genealogiis, einander zuwieder wären".

${ }^{49}$ No deja de resultar interesante que en esa misma iglesia, aunque lo diga una nota de copista no exenta de sospecha, se habría encontrado una copia de Origo; véase Socas, «L'Origo», p. 216, n. 16.

50 Kemény, Joseph. «Die Verkettung des einstens in Deutschland und Siebenbürgen in der Gestalt eines angeblichen Chripto-Socinianismus nicht lange bestandenen, sogenannten Judaismus», Magazin für Geschichte, Literatur und alle Denk- und Merkwürdigkeiten Siebenbürgens, 2, 1846/47, pp. 416429; en pp. 423-424: Magnifico et Illmo viro, ac Summo Cancellario Simoni Pechy Joannes Avitus S. P. D. Rediit ad nos ante mensem, post tot viarum ambages et tot discrimina rerum Joachimus Rasoribus, Tuaque retulit nobis non minus pientissima, quam et laudanda vota, quibus erga veram, omnique fuco liberam, ac puram veri, soliusque Dei fidem duceris, meminitque impensis verbis desiderium tuum obtinendi illius Manuscripti, cujus principia Tua esse, eaque Te perseveranter retenturum, ex interno convictus polliceris. Et ecce supervenit et alter tuus Zegedinus, juvenis sane non modestissimus solum, sed et non mediocriter in bonis litteris versatus, medio cujus iterum iterumque copiam Manuscripti Martino-Seideliani, Fundamenta Religionis Christianae inscripti, Tibi transmitti urges et sollicitas. Est ea quidem laudanda Tua diligentia, qua Te in religione, quam jam Tuam fecisse scribis, consolidare quasi intendis. At res haec plena est periculi, noris namque optime, quanta 
En su carta, Vogel accede con reticencias a la petición de Péchi de hacerle llegar a través de dos mensajeros de confianza "el manuscrito de Martin Seidel Fundamenta religionis christianae". El alemán encarece en términos sorprendentemente elogiosos la decisión de Péchi de guiarse por los principios de dicho manuscrito. Por lo demás, hace alusión a los acontecimientos ocurridos en Altdorf apenas cinco años antes y confirma su quema pública en 1616 en tanto que libro "criptosociniano". El envío, según relata Vogel, se preparó con precaución: se colocaron las páginas del manuscrito en orden inverso y se eliminó el título y el nombre del autor. 51

Entre los socinianos de Altdorf, pues, Johann Vogel es quien figura más directamente relacionado con Seidel y en concreto con el tratado hoy conocido como Origo, un texto del que, al parecer, llegó a tener al menos dos copias: la que entregó a Jacob Martini y la que años más tarde envió a Simon Péchi. En cuanto a cómo llegó el manuscrito a manos de Vogel, Zeltner especula que lo consiguió en su viaje por Polonia anterior a 1615 y en concreto en el centro sociniano de Śmigiel (Schmiegel);52 en todo caso, como destaca Heberle, 53 se encuentran evidencias de que antes del viaje de Vogel el pensamiento de Seidel expresado en Origo era conocido por otros miembros de la comunidad secreta de Altdorf; en concreto se encuentran estas evidencias en la correspondencia del referido Martin Ruar.

En una carta dirigida a Joachim Peuschel (1592-1663), otro de los principales estudiantes "cripto-socinianos", Martin Ruar se refiere a la exégesis bíblica de Martin Seidel, a quien considera "sin duda un enemigo manifiesto de nuestro Rey, y por ello detestable, pero que sin embargo a menudo se desenvolvió con no poco acierto en la interpretación de lo que llaman el sentido literal del Antiguo

asperitate, quanto fervore, quantaque importunitate his in oris, non ita diu adhuc omnes illi prosecu$t i$, quin et carceribus (quorum malorum ea tempestate et ego particeps fueram) traditi fuerrint [sic], qui similia tentasse videbantur. Terret nos sane haec praeteritarum calamitatum memoria, et quo diligentius votis tuis satisfacere cupio, eo major est in me resensus aliquid audendi, quod olim jam adeo funestos post se traxerat eventus. Interim tamen, ne desiderium Tuum hac in parte neglexisse videar, tradidi petitum opus (cujus autographum quinque abhinc annis Norimbergae, qua scriptam [sic], ut ab omnibus dici solet Chripto-Socinianum, flammis publice injectum erat), suppresso tamen tam ejus titulo, quam et auctoris nomine, paginasque inverso ordine conglutinando, Zegedino Tuo, qui peractis jam his negotiis, fidissima sub comitiva, hodierna luce ad Te, patriosque Tuos lares revertitur. Ex sincero, de caetero, annimi [sic] cordisque affectu Superos exoro, ut juvenis hic, quem laudabilis, taciturnaque sua praemissis commendare videtur modestia, iter suum felicibus omnino avibus peragere, salvusque et incolumis (de quo Te jam prospexisse scribis) domum reverti queat, ardentissima Tibi pro incolumitate felicitateque Tua meo nomine allaturus vota et desideria. Wittebergae, ubi breves solum intendo nectere moras. VII Kalend. Aug. 1621.

51 Dán, «Seidel's Origo», se dedica a este interesante caso de recepción frustrada de Origo en Transilvania, estrechamente ligada a intereses políticos. Se conoce a Péchi por sus ideas "sabatarianas" y su política de acercamiento al imperio turco. El mismo año en que Vogel escribió esta carta, el político húngaro fue encarcelado por alta traición.

52 Zeltner, Historia, I, 269, n. b.

53 Heberle, «Über den Verfasser», p. 191. 
Testamento". ${ }^{54}$ Esta carta no está fechada y de su texto sólo puede inferirse que se escribió en Racovia (Raków), el principal centro académico sociniano, del que Martin Ruar llegaría a ser rector. En las primeras páginas Ruar cuenta su viaje hasta esa ciudad polaca y no hace mención a nada que haga pensar que la "conspiración" sociniana de Altdorf ya se había descubierto, más bien al contrario. Debido a éste y a otros detalles circunstanciales, Zeltner conjetura que la carta se escribió "antes de la Pascua del año 1614", 55 y que se envió no a Altdorf sino a Jena, donde se encontraba entonces Peuschel. En el cierre Ruar manda saludos a una serie de personas, entre ellas a Johann Vogel "y también a Seidel, si se da la ocasión": pese a la sorpresa que pueda producir en primera lectura, el saludo debe referirse a Georg, no a Martin Seidel, como advierte el mismo Zeltner. ${ }^{56}$

La primera vez que Ruar menciona a Martin Seidel en esta carta lo introduce como Seidelius ille, con lo que da a entender que se trata de un referente conocido. Ruar refiere con aprobación, aunque por supuesto con matices propios, la exégesis seideliana de Isaías 7 y 8 (el niño que se llamará Emmanuel); 57 también con aprobación se refiere a la interpretación de Seidel de Isaías 53 (el "varón de dolores", etc.); 58 por último, Ruar menciona igualmente con aprobación la exégesis que hace

54 En Zeltner, Historia, II, pp. 447-484. La misma carta se había publicado anteriormente en Ruar, Martin. Martini Ruari nec non aliorum illustrium, spectabilium doctorumque virorum, quorum nonima in indice videbis, ad ipsum vel ejus causa scriptarum epistolarum selectarum centuria altera et ultima. Amstelodami: apud Davidem Ruarum, 1681, pp. 93-139. El pasaje citado se lee en Zeltner, Historia, II, pp. 464-465; más adelante (n. 59) se reproduce en su contexto.

55 Zeltner, Historia, II, p. 484. En cuanto a la fecha, debe advertirse una aparente contradicción: a lo largo de su carta Ruar hace varias referencias al De tribus Elohim de Jacob Martini, en concreto a su refutación de la interpretación del Salmo 110 como no referido en exclusiva a Cristo. El propio Zeltner (p. 465) identifica este pasaje con el correspondiente de Martini, De tribus Elohim liber tertius, p. 671 y ss.: ahora bien, dado que este Liber tertius es de 1619, la carta debería datarse forzosamente después de esa fecha, después por tanto del escándalo de Altdorf (1615), algo que sin embargo no cuadra con las circunstancias que refleja la carta. El problema se resuelve al advertir que ya en el primer volumen del De tribus Elohim, de 1614 (Martini, Jacob. De tribus Elohim liber primus, Photinianorum novorum et cum primis Georgii Eniedini blasphemiis oppositus, [Wittenberg]: Schurer, 1614, p. 260 y ss.) se encuentra una refutación de esa interpretación de ese Salmo 110, no en relación a Seidel sino a Enyedi. Ruar menciona el pasaje de Martini como paginas aliquot libri sui de tribus Elohim (Zeltner Historia, II, p. 465), sin especificar volumen, lo que invita a pensar que entonces el primer volumen era el único existente.

56 Salutem etiam transcribite Richtero meo, Planero meo, et illi meo Vogelio, et Seidelio quoque, si dabitur occasio, en Zeltner, Historia, II, p. 484 (su advertencia, en nota ahí mismo).

57 Zeltner, Historia, II, p. 461: constat enim puerum istum, qui septimo, ut et octavo capite Emanuel appellatur, esse Prophetissae filium, si sensum historicum spectes, quem Seidelius ille meo judicio non male, si modo recte memini (nam tu verba illius non adscripseras in ea charta, quam abeunti dederas) evolvit.

58 Zeltner, Historia, II, p. 462-463: Ego alias non difficulter adduci possim, ut Seideli sententiae subscribam, qui de Jeremia interpretatur, nec objici tamen posse quaedam, ignoro, quae non ita scite Jeremiae aptari possint, quod caeterorum fortassis vaticiniorum divinorum more possis excusari, quorum hoc est ingenium, ut nunc ei, ad quem mystice referunt, perfectissime conveniant... 
Seidel del Salmo 110, que la interpretación cristiana tradicional entiende como dirigido a Cristo. 59 En este último pasaje, Ruar alude a otro salmo que también "adujo para sí Seidel"; la cita permite comprobar que se trata de Salmos 21, 1. Pues bien, tanto este pasaje como todos los demás referidos de Salmos e Isaías están ausentes en el intercambio epistolar de Seidel y Sozzini, pero se encuentran interpretados y discutidos en el texto de Origo que conocemos. ${ }^{60}$ Martin Ruar, por tanto, al igual que su interlocutor, Joachim Peuschel, debía conocer al menos otro texto de Seidel diferente al de las cartas, con alta probabilidad una versión de Origo. Ruar, sin embargo, no identifica ese texto con un título ni lo cita literalmente, sino que alude a él de memoria, como se infiere del primer pasaje referido ("si es que lo recuerdo bien, pues no copiaste sus palabras en el papel que me diste al marcharme").

Así pues, Seidel era una referencia conocida entre los "cripto-socinianos" de Altdorf, y no sólo por su famoso intercambio epistolar con Sozzini, sino también por el texto Origo et fundamenta religionis christianae (con seguridad en el caso de Vogel, con elevada probabilidad en el caso de Ruar y Peuschel).

\section{Conclusiones}

Martin Seidel nació en torno a 1545, según se infiere de la fecha de su matrícula en la Universidad de Heidelberg (4 de mayo de 1564); no se encuentran indicios sobre la fecha de su muerte, más allá de la necesidad de que ésta se produjera después de su intercambio epistolar con Fausto Sozzini, que se llevó a cabo en algún momento de la década de 1580, o quizás de la de 1590.61 Zeltner escribe que Martin

\footnotetext{
59 Zeltner, Historia, II, p. 464-466: Nono quaeris, an CX psalmus de alio quam de Christo intelligi possit? Ego quidem ferme persuasus sum, posse. De quonam? dices; Judaei nonnulli de Abrahamo, nonnulli de Salomone capiendum volunt, quorum sententias ut persequar aut refellam cum aliqui somni aut olei jactura, non est mihi $<.>$ nunc tanti sufficiat si meam exposuero, in qua iterum calculum meum adjicio Seidelio, homini quidem hosti regis nostri manifesto, et ob id [p. 465] detestabili, sed qui tamen in literali, quem vocant, antiqui foederis sensu enodando non infeliciter saepe versatus est; nec tam ipsi tamen, quam aliis et pietate et eruditione praestantissimis viris, qui psalmum illum a populo de Davide cantatum non inficiantur. [...] Haec tantum contra Seidelium annotare lubet, quanquam psalmus hic a populo de Davide cantatus concedatur, nihil tamen falsi dixisse Christum nostrum affirmantem, quod David in eo Christum appellaverit Dominum suum, cum et verissimum, et apud istos Judaeos, si non recentes, saltem vetustiores illos, in confesso sit, unius loci genimum posse dari sensum [...] [p. 466] [...] annon, ut assumam exemplum ex uno psalmorum, quos pro se Seidelius hic adduxit, vere possim affirmare, Davidem dicere, "Exultabimus in salute tua, et in nomine Dei nostri vexillum elevabimus", etiamsi David populum haec dicentem introducat?

60 Lo señaló ya Heberle, «Über den Verfasser», p. 187. Heberle asume ahí mismo que la carta de Ruar se escribió en 1615, pero se basa para ello en la edición de 1684, donde sin embargo no se da fecha alguna.

61 Roth consigna así las fechas: "Martin Seidel (um 1540 - nach 1587)" (Roth, Udo. «Ut ars nostra sine philosophia doceri non possit: Der Altdorfer Mediziner Kaspar Hofmann (1572-1648)». En: Marti
} 
Seidel, tras este acercamiento frustrado a los unitarios polacos, volvió a su país y murió como maestro de escuela; pero ello no pasa de poco probable suposición, como ya señaló Heberle. 62

Martin tuvo un hermano, Jacob Seidel, probablemente algo más joven que él, que estudió con él en Heidelberg y estuvo implicado en los sucesos que rodearon su marcha de la ciudad en 1573. Jacob murió como profesor de medicina en Greifswald en 1615 y fue quizás padre de Georg Seidel; éste se matriculó en la Academia de Altdorf en 1610 y formó después parte de la comunidad de estudiantes "cripto-socinianos".

Los turbulentos años de la década de 1560 en Heidelberg constituyen el marco para la gestación de las ideas religiosas del joven Martin Seidel. Gracias a los materiales procedentes de los los Archivos de Heidelberg publicados por Hautz a mediados del siglo XIX, consta que Seidel intentó difundir en 1573 un manuscrito propio en el que negaba la naturaleza mesiánica de Cristo y que sus ideas en este sentido eran objeto de sospecha desde cinco años antes; el radicalismo crítico de Seidel es, por tanto, estrictamente paralelo en el tiempo y en el espacio al antitrinitarismo clandestino de los ministros Sylvan, Neuser y Vehe. Sin embargo, la modesta fama del silesio no se debe a su asociación con estos antitrinitarios alemanes, sino con los unitarios polacos y Fausto Sozzini, a los que intentó aproximarse tras su fracaso en Alemania. Sus cartas con el fundador del socinianismo ( $c a$. 1584), que también contienen referencia a un manuscrito propio en el que exponía sus creencias, se publicaron en varias ocasiones entre las obras de Sozzini (por primera vez en 1611); ello aseguró para la posteridad el recuerdo de las ideas de Seidel sobre religión, principalmente entre los propios socinianos y entre los protestantes que acometieron la refutación de éstos.

El socinianismo clandestino de Altdorf (1605-1617) constituye el marco en el que por primera vez se encuentra noticia expresa a un manuscrito titulado Fundamenta (o bien Origo et fundamenta, o bien Orthodoxum fundamentum) religionis christianae. El teólogo luterano Jacob Martini, que escribió una extensa refutación del mismo (1619), lo atribuye a un autor anónimo, pese a que sí conocía el nombre de Seidel, a quien cita en relación con su correspondencia con Sozzini. En cambio, el sociniano Johann Vogel, responsable de que el manuscrito llegara al conocimiento de Martini, lo atribuye a Seidel, si bien su testimonio se conoce por

\footnotetext{
- Marti-Weissenbach [eds.], Nürnbergs Hochschule in Altdorf, pp. 67-97 [p. 71]); sin embargo, se basa para ello en Schröder, «Religionsphilosophie», p. 163, quien toma como referencia para la fecha de nacimiento la supuesta expulsión de Heidelberg según Dán, Matthias Vehe, pp. 17, 177, y para la de muerte (1587) una nota de copista referida al supuesto hallazgo de un manuscrito de Origo, un dato que resulta indiferente para esta cuestión.

62 Zeltner, Historia, I, p. 270: Quo pacto domum reversus, incertum tamen, an unquam ad meliora conversus, inter pueros, quos primis elementis imbuit, consenuit, atque miser tandem animam efflavit. Véase Heberle, «Über den Verfasser», p. 191.
} 
dos documentos sólo conservados en reproducciones muy posteriores a los sucesos, de 1715 (Gundling) y 1846 (Kemény). Por lo demás, la refutación de Martini contiene la reproducción de extensos pasajes del manuscrito que confirman su elevada semejanza textual, aunque no su identidad, con las versiones del mismo que se conocen actualmente. ${ }^{63}$ Una de las cartas de Martin Ruar a Joachim Peuschel ( $c a$. 1614) confirma que ambos conocían un texto de Seidel en el que se interpretaban determinados pasajes bíblicos del mismo modo a como se encuentran interpretados en las versiones de Origo que conocemos.

Resulta interesante observar cómo el pensamiento de Seidel nace y se desarrolla en el contexto de la Reforma protestante radical de finales del siglo XVI, para ingresar más tarde en el corpus de tratados filosóficos clandestinos del siglo XVIII. Esta evolución se debe probablemente al carácter plus quam haereticus (como lo llamó Zeltner) de nuestro autor; del mismo modo puede achacarse a este carácter su lugar hasta ahora marginal en la historia de las ideas: por un lado, sus creencias, que niegan la revelación cristiana y consideran extinta la revelación judía, trascienden la "herejía" y son extrañas al reformismo cristiano de su época; por otro lado, su discurso, que de acuerdo con su tiempo se apoya ante todo en la interpretación bíblica, desentona en el cuadro general de los tratados filosóficos entre los que circuló un siglo más tarde.

Pablo Toribio Pérez

Departamento de Filología Griega y Latina

Universidad de Sevilla

pablotoribio@us.es

63 Ejemplos en Heberle, «Über den Verfasser», pp. 184-186. Las copias conocidas de Origo en Benítez, Miguel. La face cachée des Lumières. Paris - Oxford: Universitas - Voltaire Foundation, 1996, p. 44. 